\title{
Desmaterialización de valores mobiliarios: Algunas reflexiones a propósito de la Ley de Títulos Valores
}

Amílcar Adolfo Mendoza Luna

"Lo común a todas estas transacciones es que todo lo que circula en ellas son impulsos electrónicos, radicalmente intangibles. El oro reemplazó en una época a las mercancías; a su vez fue sustituido por el papel (billetes, acciones, letras, bonos, etc.); ahora éste es sustituido por simples pulsos electrónicos. Vamos pues hacia el fin del papel moneda, a una gran aceleración de las transacciones, que se acercan al tiempo real, a una significativa pérdida del poder de los bancos y hacia la emergencia de nuevas formas de contabilidad social del trabajo".

Nelson Manrique. La sociedad virtual y otros ensayos ${ }^{1}$.

\section{Introducción}

En los albores de la humanidad, las normas de convivencia social se fundamentaban en la costumbre, la cual gozaba de mayor validez en tanto era más antigua. Con el correr del tiempo y de acuerdo a las necesidades sociales y económicas el derecho pasa de ser un conjunto de fórmulas rituales a un sistema apoyado en documentos escritos. Es muy conocida la frase: «lo que no está escrito en el papel no existe para el derecho.»²

1 Nelson Manrique, La sociedad virtual y otros ensayos. Lima: Fondo Editorial PUCP. 1997. p.44.

2 Ver Fernando de Trazegnies Granda, «La desmaterialización del derecho. Del derecho de pernada al internet». En: Thémis No 38. pp. 7-14. Trazegnies a partir del «derecho de pernada" medieval y los ritos formales del derecho romano entre otros, nos comenta 
En nuestra opinión, existe lo que se denomina «dependencia del papel» ${ }^{3}$. El papel es el soporte documental utilizado con mayor intensidad hasta estos años a pesar de las desventajas que tiene, las cuales son fáciles de encontrar, un papel se puede deteriorar o extraviar y si son muchos se necesita a veces un experto para clasificarlos y mantenerlos ordenados, sin olvidar que su contenido se puede falsificar o adulterar. Para decirlo en una palabra, es un medio "análogo»: conforme se use una y otra vez sufrirá un deterioro. Además no siempre se obtendrá una reproducción perfecta (lo cual ocurre con las fotocopias o las reproducciones de un cassette). La información sería aproximada.

"La información analógica se puede recopilar, almacenar y reproducir, pero tiende a ser imprecisa y corre el riesgo de hacerse menos precisa cada vez que se transfiere». ${ }^{4}$

como los signos exteriores de publicidad de los actos jurídicos empiezan a convertirse en intangibles. En nuestra opinión se refiere a un tema distinto al que ahora nos ocupa. Se refiere a la dicotomía planteada entre los signos del mundo físico (análogo) y el mundo digital. La desmaterialización de valores es sólo un aspecto de un fenómeno mayor que abarca el dinero, los contratos y todo lo que pueda reposar en un documento o ser materia de un acto jurídico.

3 Puede encontrarse una reflexión mayor sobre este tema en: Amílcar Adolfo Mendoza Luna. «Desmaterialización de Valores mobiliarios: Rol de CAVALI ICLV S.A. en el mercado de valores peruanon. Tesis (PUCP) para optar por el título de abogado. 1998, pp.7990. También Rolando Castelares Aguilar, Los documentos y títulos valores electrónicos. Su normatividad legal en el Perú. Lima: Mac Editores. 1992. p.23; Alain Salomon, "Desmaterialización de los valores mobiliarios franceses». En: Revista del Instituto Argentino del Mercado de Capitales. Año 2. No 6. Enero-abril 1987. Alain Salomón (en la fecha de redacción del artículo era Director General de SICOVAM) se plantea la pregunta de cómo inmovilizar el papel, o mejor dicho como poder administrarlo o incluso eliminarlo de las transacciones y menciona el objetivo del mercado de valores de llegar a una sociedad sin papel «certificateless society». P. Dominguez Sors, «El nuevo sistema de liquidación y depósito. Comentarios al informe de la comisión». En: Boletín Financiero de la Bolsa de Barcelona. Julio 1979. $3^{\circ}$ época. Año XVIII. Núm.70. El autor recuerda la caótica situación enfrentada ante una fuerte contratación bursátil sobre títulos físicos paper crunch. William H Gates III, Camino al futuro. New York: Penguin Books, 1995. p. 11 1; Héctor Alegria,. La desmaterialización de los títulos valores. En: Revista Peruana de Derecho de la Empresa. Lima. No 35 (Octubre 1989). pp. 55-60. De manera clara y precisa se destaca la situación producida por la dependencia del papel y el descontrol jurídico que produce. Parafraseando a René Roblot nos indica que «asistimos en cien años a la grandeza y decadencia de la noción de título físico" y que hay una revolución que amenaza desaparecer al soporte papel. 
Hagamos un esfuerzo de imaginación y representémonos la cantidad gigantesca de valores mobiliarios que se transfiere en nuestra Rueda de Bolsa y en las del extranjero. El día que escribimos estas líneas, 23 de abril, existen en circulación 1,502'330,834 acciones de Telefónica B. y no contamos los demás valores listados, ni mencionamos aún que nuestra bolsa es considerada mediana en el ámbito latinoamericano.

Administrar grandes cantidades de acciones representadas en títulos físicos sería complicado. Involucraría ineficiencias como los notables costos de custodia y logística para transferir dichos certificados a sus nuevos titulares.

A lo largo de este artículo explicaremos brevemente algunas de las ventajas más relevantes de la desmaterialización de valores mobiliarios para efectos de su negociación en Rueda de Bolsa. Para ello explicaremos que la desmaterialización es la culminación de un proceso que tiene sus raíces en los sistemas de depósito que usaban el método de anotación en cuenta (lo cual nos remontará a finales del siglo XIX) y en el fenómeno informático frente al desafío de la dependencia del papel. De esta manera podrá apreciarse como el soporte informático (técnica digital) es una alternativa al soporte papel (técnica análoga) que puede superarlo en confrabilidad y exactitud 5 .

\section{Las anotaciones en cuenta y los sistemas de deposito colectivo de valores mobiliarios}

Según José Murua ${ }^{6}$ existen tres formas conocidas de depósito de valores mobiliarios:

4 William H. Gates IHI, op. cit. pp.21-29. Es interesante y sencilla la manera como Bill Gates nos explica la diferencia entre el medio digital y análogo a través del ejemplo de las distintas iluminaciones que se pueden obtener graduando focos de luz. Por razones de espacio y por ser un tema técnico preferimos recomendar la lectura de este autor.

5 «[...] principalmente en las últimas décadas, la humanidad ha logrado grandes adelantos tecnológicos que, como no podía ser de otro modo, han incidido en los tradicionales conceptos jurídicos en los que descansaba el derecho cambiario, obligándonos a revisarlos y postular nuevas visiones y conceptos, sin afectar en su esencia los principios jurídicos antes señalados, aún cuando el soporte no sea necesariamente el papel sino el electrónico o desmaterializado" (Exposición de motivos de la Presentación del Proyecto de Ley de Títulos Valores del 17 de junio de 1999, p.5).

6 José Murua, «El depósito colectivo de valores sobre el funcionamiento de la Caja de 
a) El depósito regular (clásico): donde los valores quedan individualizados por su cantidad y especie pero además por la denominación cuantitativa de cada lámina o certificado y numeración de los mismos. Por supuesto que el depositario tendrá que devolver exactamente el mismo documento. Lo más probable es que lo custodie en una bóveda de alta seguridad, perfectamente individualizado y sin posibilidad de confusión posible con otros.

b) El depósito irregular: donde el depositante pierde la titularidad del documento pero adquiere un derecho de crédito frente al depositario. Es similar al depósito de dinero (papel moneda). Entregamos al banco billetes que representan una cierta cantidad, pero no podemos esperar que nos devuelvan exactamente los mismos billetes.

c) El depósito colectivo: el título se convierte en fungible. No se devuelve el mismo título depositado sino otro de la misma especie y calidad. Sin embargo, los títulos físicos quedan inmovilizados y su transferencia se lleva a cabo mediante asientos contables por los cuales la entidad depositaria emite certificados. ${ }^{7}$

El sistema de depósito colectivo se usó en la práctica bancaria alemana desde 1882 cuando se fundó el «Wiener Giro und Kassen Verein». Luego de la Primera Guerra mundial se generalizó y se reguló mediante la Ley del 4 de febrero de 1937, sobre depósito y adquisición de Títulos Valores. Como los títulos quedaban inmovilizados y las transferencias se hacían con la simple anotación en el libro de Registro de Depósitos del banco no hay manipulación material de los títulos. Para prever la transferencia entre banco y banco se reguló la centralización de los depósitos en bancos colectores" (un depósito de segundo grado). Los bancos perdían la posesión mediata de la cartera de depósitos y la pasaban a los bancos colectores. $^{8}$

Valores en Argentina». En: Asamblea General de la Federación Iberoamericana de Bolsas de Valores. Ciudad de México D.F. Noviembre 1976, p.99.

7 Cabe aclarar, aun cuando este detalle se advertirá más adelante, que esta anotación en cuenta es previa a la desmaterialización, la cual involucra la supresión total de todo certificado (título físico) y no involucra su inmovilización sino su reemplazo por un documento electrónico.

8 José Murua, op.cit. pp.99-102. La razón del auge de este sistema luego de la I guerra mundial es la hiperinflación que se sufría, la cual ocasionó que las Sociedades Anónimas quedaran representadas por gigantescas masas de títulos físicos de ínfimo valor lo cual creó una situación confusa y difícil de administrar. Los memoriosos recordaran que se vivió hace una década una situación igualmente siniestra en Perú. No sorprenderá entonces que el 
Como resultado del éxito de este sistema las transferencias se hicieron con mayor seguridad y rapidez. Durante la II Guerra mundial, los alemanes invadieron Francia e introdujeron este sistema.

La "Caisse Centrale de Depots et de Virements de Tires» - CCDVT (Caja Central de Depósitos y Transferencias de Títulos) se creó mediante Ley del 18 de junio de 1941 para aceptar en deposito las acciones al portador de las sociedades anónimas francesas por cuenta de «establecimientos afiliados" (bancos, instituciones financieras y agentes de bolsa), de esa manera se facilita la transferencia de acciones mediante transferencias de cuenta a cuenta entre estos afiliados. El trasfondo político de esta medida era que el gobierno de Vichy necesitaba el control de la actividad extranjera durante la ocupación. Teniendo en cuenta que el mercado francés tenía marcada preferencia por los títulos al portador había que vigilar las adquisiciones de alemanes, quienes podrían intentar copar las empresas francesas mediante la bolsa. ${ }^{?}$

Mediante la Ley del 3 de febrero de 1943 se impuso la obligatoriedad del sistema de anotaciones en cuenta a las acciones al portador. Luego de la guerra y pasada la amenaza germánica este sistema recibe críticas duras. Por tal motivo se creó la Societé interprofessionelle pour la compensation de valeurs mobiliéres - SICOVAM (Sociedad interprofesional para la compensación de los valores mobiliarios) el cual es un depósito facultativo. En los años 80 el porcentaje de acciones al portador que no pasaba por la SICOVAM era apenas de $5 \%$ y la emisión de títulos físicos decrecía rápidamente. Actualmente la desmaterialización en Francia es total y la SICOVAM es considerada la más avanzada entidad desmaterializadora.

En el resto de Europa podemos mencionar a:

- Deutscher Kaissenverein A.G. DKV (Alemania),

- El Servicio de Compensación y Liquidación de Valores de España ${ }^{10}$,

sistema de anotaciones en cuenta se desarrolle en plena época hiperinflacionaria en nuestro medio(1988).

9 José Murua, op.cit.pp.101-102; Daniel Espina,. Las anotaciones en cuenta. Un nuevo medio de representación de los derechos. Civitas: Madrid. 1995. pp. 134-136. Alain Salomon,. op. cit. p.28.

10 Un amplio estudio sobre la desmaterialización y la SCLV se encuentra en Daniel Espina, op. cit.pp. 142-149 y Ana Ibáñez, "Compensación y Liquidación de Valores en España». En: Valores. Revista de CONASEV. Lima: Ed. Amistad. Año VII. No 22. pp. 3035. 
- Schweizerische effekten Giro AG: SEGA (Suiza),

- Euroclear (Bélgica),

- CEDEL (Luxemburgo).

En América destacan:

- El Depositary Trust Company (DTC),

- El Instituto para el Deposito de Valores de México (SD Indeval) ${ }^{11}$,

- La Caja de Valores de Argentina,

- La Cámara de Liquidación y Custodia de Río de Janeiro,

- La Caja Venezolana de Valores,

- El Depósito Centralizado de Valores de Colombia ${ }^{12}$ y

- CAVAli ICLV S.A. de Perú ${ }^{13}$

Para sintetizar lo expuesto hasta ahora incluimos el siguiente cuadro:

\begin{tabular}{|c|c|c|c|c|c|c|}
\hline & $\begin{array}{c}\text { Valores } \\
\text { Individualizados }\end{array}$ & $\begin{array}{l}\text { Custodia } \\
\text { de Tírulos } \\
\text { físicos }\end{array}$ & Fungible & $\begin{array}{c}\text { Inmovilización } \\
\text { de Títulos } \\
\text { físicos }\end{array}$ & $\begin{array}{c}\text { Supresión } \\
\text { de Títulos } \\
\text { físicos }\end{array}$ & $\begin{array}{l}\text { Anotación } \\
\text { en cuenta }\end{array}$ \\
\hline DepósitoRegular & $\mathrm{X}$ & $\mathrm{X}$ & & & & \\
\hline Depósito Irregular & & $\mathrm{X}$ & $X$ & $\mathrm{X}$ & & \\
\hline Depósito Colectivo & & $X$ & $X$ & $\mathrm{X}$ & & $\mathrm{X}$ \\
\hline Desmaterialización & & & & & $X$ & $\mathrm{X}$ \\
\hline
\end{tabular}

Podemos ver con claridad las diferencias entre una y otra clase de depósito y además adelantamos algunas notas distintivas de la desmaterialización en la cual, si bien usa el método de la anotación en cuenta, se prescinde de todo título físico.

Héctor Alegría ${ }^{14}$ con respeto al tratamiento masivo de los títulos en serie plantea una sucesión de etapas en el tránsito hacia la desmaterialización:

a) Se fue admitiendo la emisión de títulos múltiples que eran representativos de varias unidades: en un sólo título físico se representan varias acciones.

11 consultar su página WEB http://www.bmv.com.mx/html/indevalesp.html

12 Una fuente de información rápida y sencilla sobre el sistema de depósito de Colombia se puede encontrar en: María Carolina García Guzmán, Consecuencias Jurídicas de la desmaterialización de Títulos Valores: el caso de los depósitos centralizados de valores en Colombia. Santa Fe de Bogotá D.C.: Pontificia Universidad Javeriana. 1991. pp. 68-79.

13 Recomendamos consultar la página web de CAVALI ICLV S.A. en http:// bvl.com.pe/cavali

14 Héctor Alegría, op. cit. pp.58-59. 
b) Cuando se implementaron los sistemas de gestión o depósito centralizado de títulos (que ya conocemos), los títulos múltiples dieron lugar a "certificados globales".

El certificado global era un único título físico representativo de la totalidad de la emisión o al menos de una parte importante de ella.

c) Los sistemas de depósito reciben los certificados globales y para las transferencias bastan anotaciones en cuenta. No se requería partir el título en sucesivos documentos escritos para cada titular. El título físico es aún necesario para crear el valor mobiliario, pero ya no lo es para transferirlo.

d) Cuando los sistemas de deposito omiten la presentación del título para el ejercicio del derecho y permiten anotar toda medida que afecte la titularidad (como gravámenes, embargos, transferencias) sólo bastaba el último ingrediente: la tecnología informática, para desprenderse del soporte papel y prescindir del título físico desde el origen del derecho de titularidad sobre la acción.

De acuerdo a la FIABV (Federación Iberoamericana de Bolsas de Valores) en 1994 la situación de los depósitos de valores latinoamericanos era como se indica a continuación:

\section{Regímenes de anotación en cuenta para valores mobiliarios}

\begin{tabular}{|l|c|c|}
\hline País & Inmovilización & Desmaterialización \\
\hline México & $\mathrm{X}$ & \\
Venezuela & $\mathrm{X}$ & \\
Colombia & $\mathrm{X}$ & $\mathrm{X}$ \\
Argentina & $\mathrm{X}$ & $\mathrm{X}$ \\
Chile & $\mathrm{X}$ & $\mathrm{X}$ \\
Brasil & $\mathrm{X}$ & $\mathrm{X}$ \\
España & $\mathrm{X}$ & $\mathrm{X}$ \\
Perú & & \\
\hline
\end{tabular}

Notemos que en estos países coexistía la inmovilización del título físico y la desmaterialización. Es decir; si bien se habían suprimido algunos títulos físicos y fueron reemplazados por documentos electrónicos, para algunos valores las transferencias de titularidad, entrega de dividendos, etc. se hacen mediante anotaciones en cuenta pero custodiando el título físico para efectos de poder hacer correcciones si fuera necesario. 
El tránsito a la desmaterialización total es progresivo. Se necesita confianza de los inversionistas en el sistema y un ente profesional a cargo. De esta manera, los inversionistas no tendrán incertidumbre ni se verán tentados a solicitar la reversión del proceso (es decir, pasar de la desmaterialización a tener nuevamente un título físico entre las manos).

Según Mario Segura ${ }^{15}$ la desmaterialización es la supresión físico material de los títulos, subsistiendo los derechos de cada uno de los titulares en la memoria de un ordenador electrónico, comprendiendo por memoria el archivo que de constancia de los derechos correspondientes.

"La desmaterialización, en conclusión, es el reemplazo de un objeto físico por signos electrónicos o bits en la memoria de una computadora. La desmaterialización en los mercados financieros generalmente ocurre en fases. Un certificado de acciones puede ser reemplazado por un registro contable que puede ser imprimido en un papel o mantenerse en un soporte electrónico como archivo". ${ }^{16}$

Para dar un ejemplo de las repercusiones de esta discusión doctrinaria en nuestro medio, citaremos la Exposición de Motivos de la presentación del Proyecto de la Ley de Títulos Valores (17 de junio de 1999) en la cual se explica que debido a las nuevas corrientes y la legislación comparada unánimemente se admiten y vienen adoptando cambios, haciendo posible la desmaterialización de los títulos valores. Es decir, «se prescinde del soporte papel, asegurado con sellos y firmas autógrafas -que hasta hace poco venía siendo utilizado con exclusividad-, para sustituirlo por otro que sea más seguro y confiable». ${ }^{17}$

15 Mario Segura Quiroz, "Desmaterialización de los títulos valores». En: Revista del Instituto Argentino de Mercado de Capitales. Año 2. No 8. Set-Dic. 1987. p.65. Al momento de escribir su artículo, el Lic. Segura era Director General de la Bolsa mexicana de valores.

16 Amílcar Adolfo Mendoza Luna, op. cit.p.110. No sólo las acciones, sino incluso cualquier instrumento financiero o el dinero se puede desmaterializar (se convierte en dinero digital o e-cash). Ver también: http://www.amex.com.cgi-bin "The dictionary of financial risk managements de AMEX.

17 Proyecto de Ley de Títulos Valores publicado en «El Peruano» el 17 de junio de 1999 , pp.5-6. 


\section{La informática y su aplicación en las anotaciones en cuenta}

Ha quedado establecido que la anotación en cuenta es un método que incluye a la desmaterialización y a la inmovilización de valores. Falta establecer la conjunción entre las anotaciones en cuenta y la informática (disciplina que estudia el tratamiento racional y automático de la información) $)^{18}$.

Al inicio de la década de 1980, Dinamarca y Suiza se adelantan a Francia en establecer la desmaterialización total a fin de facilitar el rápido flujo de capitales. El arma que se usaba era la transferencia electrónica de fondos (TEF) a través de computadoras.

La información comienza a tener un valor económico que no había tenido hasta entonces y se empieza a hablar de una «tercera ola» del conocimiento que superaría a la etapa industrial (segunda ola) y agraria (primera ola) de la humanidad. ${ }^{19}$

Como sabemos, los sistemas de depósitos que se limitaban a inmovilizar el título físico, si bien eliminaban el problema de la "traditio» al nuevo titular, tenían el problema de la custodia del valor mobiliario, el registro de transferencias seguía siendo manual y propenso al error humano.

Ante esta situación, la tecnología informática revoluciona la actividad humana planteando un sustituto al certificado físico de acciones, el documento electrónico. La pregunta ¿cómo deshacerse del papel? ya tenía respuesta. ${ }^{20}$

Basta con hacer un contraste para darse cuenta del tremendo cambio que involucra la desmaterialización: los pagos (liquidaciones bursátiles) se hacen en menor tiempo ${ }^{21}$. En cada operación de compraventa ya no es necesaria la manipulación de títulos físicos, tener un control manual de éstos o anotar sus numeraciones en documentos de entrega, pólizas, regis-

18 Rocío Rondinel Sosa, Informática Jurídica. De la Teoría a la Práctica. Lima: Preai S.R.L. 1995, pp.25-32.

19 Alvin Toffler, La tercera ola. Madrid: Plaza \& Janes. Traducción de Adolfo Martín. 1994 , pp. 25-33.

20 «Actualmente, en todo el mundo, los administradores de valores mobiliarios se plantean la misma pregunta: ¿̨ómo eliminar o inmovilizar el papel?» Alain Salomon, op. cit. p.27.

21 En nuestro medio es $T+3$, es decir a 72 horas de ejecutada la orden del comitente en Rueda de Bolsa. 
tros, depósitos, etc. multiplicando procesos de control y registro de cupones (como cuando se pagan dividendos).

También desaparece el riesgo de pérdida y falsificación de certificados de acciones (títulos físicos), los costos e ineficiencias que conllevan la custodia de títulos físicos (bóvedas, pólizas flotantes de seguros, sistemas de seguridad contra robo e incendios, etc.). Si hay menos dificultad en administrar los valores, se reducen los costos de transacción.

Por otro lado, encontramos un ente profesional: el sistema de depósito colectivo que asume las responsabilidades por el correcto manejo de las transferencias y registro de las titularidades.

Por el lado del inversionista; este puede comprar y vender valores en un mínimo de tiempo, evitando distorsiones en los precios generados por la demora en la entrega de los títulos físicos. Se hace más fácil para el inversionista estar el tanto de los dividendos, entrega de derechos de suscripción preferente, gravámenes o embargos que recaigan sobre sus valores. Existiendo transparencia en el mercado, se proporciona a los titulares de acciones la información periódica sobre sus tenencias pudiendo decidir rápidamente que negociar.

\section{Algunos comentarios sobre las anotaciones en cuenta y la desmaterializacion en la legislación peruana}

En la doctrina de la desmaterialización existe una pregunta que es la primera que debe resolver cada legislador: ¿se aplica solamente a los títulos individuales o a aquellos que se emiten en masa?.

En la actualidad, se desmaterializan los valores emitidos en masa como las acciones o los bonos, ¿podrían ser desmaterializados los cheques u otros títulos como ocurre en Francia con la "Lettre de change relevé»?.

Cuando la legislación se ocupa de los valores mobiliarios se refiere a todos aquellos valores que cumplen con la condición de ser emitidos en forma masiva, son libremente negociables y confieren a sus titulares derechos crediticios, dominiales o patrimoniales, o los de participación en el capital, el patrimonio o las utilidades del emisor (Art. $3^{\circ}$ Decreto Legislativo No 861, Ley de Mercado de Valores).

Los valores mobiliarios pueden representarse por anotaciones en cuenta o títulos físicos a voluntad del emisor (Art. 209\% LMV y Art. $42^{\circ} \mathrm{del}$ Reglamento de Instituciones de Compensación y Liquidación de Valo- 
res, aprobado por la Resolución CONASEV No 031-99-EF/94.10, publicada el 5 de marzo de 1999 en "El Peruano»). Cualquiera que sea su forma de representación confieren los mismos derechos y obligaciones a sus titulares (Art. $80^{\circ} \mathrm{LMV}$ ).

Hay que notar que la legislación peruana sólo trata el tema de las anotaciones en cuenta para los valores mobiliarios (como las acciones o bonos) dentro de la legislación del mercado de valores. Incluso la nueva Ley General de Sociedades (Ley No 26887 publicada el 9 de diciembre de 1997 en "El Peruano») al referirse a la Matrícula de Acciones (Art. 920) claramente indica que el régimen de representación de valores mediante anotaciones en cuenta se rige por la legislación del mercado de valores.

Cabe anotar que a través de la Ley de Títulos Valores por primera vez se incorpora en nuestra legislación el término desmaterialización, aunque sin mencionar una definición. En este artículo hemos ofrecido una primera aproximación a este término que es nuevo en la doctrina y debe ser objeto de discusión.

Nos parece que en la forma como está planteada la cuestión en la Ley de Títulos Valores, la anotación en cuenta (desmaterialización) regiría para los valores mobiliarios emitidos en masa. La razón principal de esta decisión radicaría en que cuando tenemos títulos singulares no existe el elemento de fungibilidad ni la posibilidad de administración por un sistema de depósito, a menos que surja algún servicio similar a través de la práctica bancaria.

En la Exposición de Motivos del Proyecto hay un notorio interés por encontrar una denominación que sea más apropiada para la realidad actual:

"Así, habiéndose identificado lo materializado con el título (papel) y lo desmaterializado (sin papel, electrónico) con un mero registro en cuenta, resulta impropio seguir denominando "Títulos" valores a esta categoría de documentos comerciales que no siempre tienen una representación materializada. Por ello, se propone que los títulos valores con aptitud o posibilidad de circulación o transmisión, a los que en la doctrina y legislación comparada se viene denominando "valores negociables", tenga esta misma denominación genérica y que, a su vez, pueden constituir valores en "título" (cuando el valor sea materializado o tenga soporte papel) y valor "con representación por anotación en cuenta" (cuando el valor tenga soporte electrónico o conste en un registro).» 
De acuerdo a lo anterior, la nueva Ley de Títulos Valores regula tanto a los títulos físicos como a los desmaterializados, porque la única diferencia relevante entre ellos es el soporte utilizado, lo que no altera su naturaleza jurídica ni económica.

\section{A modo de conclusión}

La desmaterialización de valores mobiliarios es el resultado de la fusión de dos procesos: la anotación en cuenta y el auge de la informática. Si bien no es un concepto proveniente del derecho, sino la reacción del tráfico mercantil ante la dependencia del papel; existen señales de recepción de este fenómeno por parte del derecho. Por ahora, la encontramos asociada a los valores emitidos en masa y en lo que se pueda adaptar a otros títulos valores de acuerdo a la Ley actual. En todo caso, aún se discute en la doctrina si la desmaterialización se puede aplicar a otros títulos valores que no sean aquellos emitidos en masa manteniendo las mismas ventajas de fluidez en el tráfico y seguridad jurídica. 